\title{
Imaging in the Evaluation of Endoscopic or Surgical Treatment for Achalasia
}

\author{
Diego Palladino, ${ }^{1}$ Andrea Mardighian, ${ }^{2}$ Marilina D'Amora, ${ }^{3}$ \\ Luca Roberto, ${ }^{3}$ Francesco Lassandro, ${ }^{4}$ Claudia Rossi, ${ }^{3}$ Gianluca Gatta, ${ }^{3}$ \\ Mariano Scaglione, ${ }^{5}$ and Guglielmi Giuseppe ${ }^{2}$ \\ ${ }^{1}$ Radiology Department, IRCCS “Casa Sollievo della Sofferenza”, San Giovanni Rotondo, Foggia, Italy \\ ${ }^{2}$ Radiology Department, University of Foggia, Foggia, Italy \\ ${ }^{3}$ Radiology Department, Second University of Naples, Napoli, Italy \\ ${ }^{4}$ Radiology Department, "V. Monaldi" Hospital, Napoli, Italy \\ ${ }^{5}$ Radiology Department, Pineta Grande Medical Center, Castel Volturno, Caserta, Italy
}

Correspondence should be addressed to Gianluca Gatta; ggatta@sirm.org

Received 19 June 2015; Accepted 13 September 2015

Academic Editor: Lorenzo Mannelli

Copyright (c) 2016 Diego Palladino et al. This is an open access article distributed under the Creative Commons Attribution License, which permits unrestricted use, distribution, and reproduction in any medium, provided the original work is properly cited.

\begin{abstract}
Purpose. Aim of the study is to evaluate the efficacy of the endoscopic (pneumatic dilation) versus surgical (Heller myotomy) treatment in patients affected by esophageal achalasia using barium X-ray examination of the digestive tract performed before and after the treatment. Materials and Methods. 19 patients (10 males and 9 females) were enrolled in this study; each patient underwent a barium X-ray examination to evaluate the esophageal diameter and the height of the barium column before and after endoscopic or surgical treatment. Results. The mean variation of oesophageal diameter before and after treatment is $-2.1 \mathrm{~mm}$ for surgery and $1.74 \mathrm{~mm}$ for pneumatic dilation (OR 0.167, CI 95\% 0.02-1.419, and $P: 0.10$ ). The variations of all variables, with the exception of the oesophageal diameter variation, are strongly related to the treatment performed. Conclusions. The barium X-ray study of the digestive tract, performed before and after different treatment approaches, demonstrates that the surgical treatment has to be considered as the treatment of choice of achalasia, reserving endoscopic treatment to patients with high operative risk and refusing surgery.
\end{abstract}

\section{Introduction}

Achalasia is the most frequent primary motor disorder of the esophagus. It is still a rare disease that may occur in both sexes at any age with a prevalence of less than $1 / 10,000$ and with a new cases' incidence of $0.6-1 / 100,000$ citizens/year [1]. At the base of this disease there is a primitive neuromuscular alteration with a myenteric plexus degeneration causing a pathophysiological disorder consisting in the failure of the lower esophageal sphincter (LES) relaxation during swallowing and the complete loss of peristaltic coordination of the esophagus body [2]. Dysphagia is the typical symptom, consisting in the difficulty in swallowing food; usually the patients have a very long and often unrelated history [3]. Other times the patients may show a sudden onset and, rarely, the symptomatology may be "paradoxical," more pronounced for liquids than solids. Patients may also feel chest pain and regurgitation. Pain is a less frequent symptom and it is usually observed in the early stages of the disease. It is probably related to the smooth muscles contraction of the esophageal body. Regurgitation is the symptom occurring in later stages, when the esophagus is dilated, and may be misdiagnosed as a gastroesophageal reflux disease, leading to diagnosis delay. In this phase, aspirations of food material may be also present leading to "ab ingestis pneumonia" in $12 \%$ of cases [1]. Other times, finally, the only sign of this disease can be a persistent halitosis, due to stagnation of endoesophageal food material. The diagnosis is usually made with $\mathrm{X}$-rays of the digestive tract with barium contrast medium $(\mathrm{cm})$ administration and esophageal manometry [4]. The therapeutic approach may be pharmacological, endoscopic, and surgical [5]. 

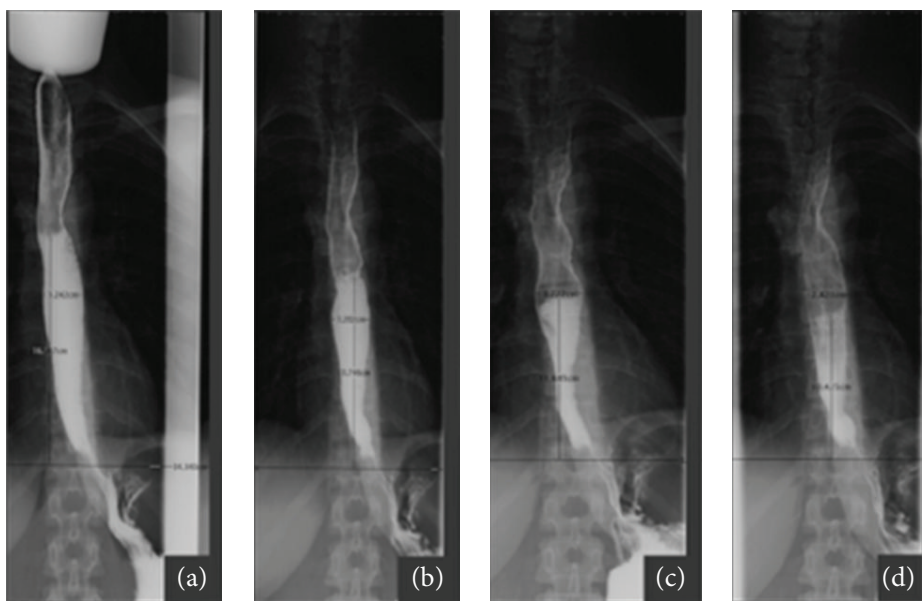

FIGURE 1: X-rays show the height of barium column $0,1,2$, and 5 minutes after barium oral administration.

Aim of the study is to evaluate the efficacy of the endoscopic (pneumatic dilation) versus surgical (Heller myotomy) treatment in patients affected by esophageal achalasia through the analysis of parameters deriving from the barium X-ray examination, performed before and after surgical or endoscopic treatment.

\section{Materials and Methods}

The study was approved by the Institutional Ethical Committee and conducted according with the ethical principles of the Declaration of Helsinki. Written informed consent was obtained in all patients.

From January 2009 to December 2014 all the patients referring to our radiology departments for radiological evaluation of achalasia, based on previous esophageal manometry, and planned for surgical or endoscopic treatment were investigated about their clinical history and eligible patients were considered for enrolment in this study. Patients with concomitant systemic neurological and/or rheumatologic disease (e.g., Parkinson disease, scleroderma) were excluded.

Each patient underwent barium X-rays evaluation before and after endoscopic or surgical treatment.

The examination was performed with Siemens AXIOM Luminos DRF equipment. Pronto Bario HD (Bracco, Milan, Italy) has been used as contrast medium; each patient received $98,45 \mathrm{~g}$ of powder for oral suspension diluted in $90 \mathrm{~mL}$ of water and administered as a single bolus prior to the execution of swellings at $0,1,2$, and 5 minutes (Figure 1). The esophageal diameter and the height of the barium column were evaluated at the different times after the barium administration. The procedure was successful and was well tolerated in all patients and no complications were reported during the exam execution.

2.1. Statistical Analysis. To obtain the logistic model we calculated, for each variable, the mean change was observed after the intervention of the total sample. For each patient were introduced five dichotomous variables (one for each initial variable), assuming value 1 if the reduction found in a particular patient results to be greater than or equal to the average reduction and 0 otherwise. Finally, for each variable, we evaluated the possible relationship with the treatment and we calculated odds ratio.

\section{Results}

Fifty-two patients were initially considered for the study, 12 patients were excluded due to the presence of concomitant systemic neurological disease, 17 did not give their consent, and 4 patients were lost to the evaluation after treatment. Nineteen patients were finally enrolled: 10 males and 9 females, age range was 27-76 y.o. for men and 41-75 for women. Eleven patients underwent surgical Heller myotomy treatment and Dor fundoplication and 8 had endoscopic pneumatic dilation treatment performed, due to the high operative risk and refusal of surgical treatment.

The mean variation of esophageal diameter before and after treatment is $-2.1 \mathrm{~mm}$ for surgery and $1.74 \mathrm{~mm}$ for pneumatic dilation (OR 0.167, CI 95\% 0.02-1.419, and $P$ : $0.10)$. Table 1 shows the variation of esophageal diameter and the height of barium column before and after surgical or endoscopic treatment at $0,1,2$, and 5 minutes after barium administration. Table 2 shows the odds ratio calculated with the logistic regression model to demonstrate postoperation mean changes in relation to the two treatments.

\section{Discussion}

The standard in diagnosing and classifying achalasia is represented by the esophageal manometry documenting the impaired relaxation of the LES and the absence or the alteration of peristaltic waves in the distal esophagus [4, 6-9].

Upper endoscopy is usually performed to rule out cancer or a peptic stricture and, particularly in patients older than 50 years with dysphagia and weight loss, attention should be paid on the possible presence of a tumor underlying achalasia (pseudoachalasia) [10, 11]. Cytohistological samples should always be taken in the cardiac region and in the suspicious areas, to find possible neoplastic degeneration $[12,13]$. Chest 
TABLE 1: Table shows the variation of esophageal diameter and the height of barium column before and after surgical or endoscopic treatment at $0,1,2$, and 5 minutes after barium administration.

\begin{tabular}{lcccccc}
\hline & \multicolumn{2}{c}{$\begin{array}{c}\text { Surgery } \\
\text { Myotomy }\end{array}$} & \multicolumn{3}{c}{$\begin{array}{c}\text { Endoscopy } \\
\text { Pneumatic dilation }\end{array}$} \\
& \multicolumn{2}{c}{ 11 patients } & \multicolumn{3}{c}{ 8 patients } \\
& Before & After & $\Delta$ & Before & After & $\Delta$ \\
\hline $\begin{array}{l}\text { Esophagus } \\
\text { diameter (cm) } \\
\text { Column baryta }\end{array}$ & 5.20 & 3.10 & -2.10 & 4.80 & 6.54 & +1.74 \\
height & & & & & & \\
$\quad 0^{\prime}$ & 23.95 & 11.9 & -12.05 & 26.50 & 14.66 & -11.89 \\
$1^{\prime}$ & 21.30 & 7.31 & -14.00 & 25.85 & 12.61 & -13.24 \\
$2^{\prime}$ & 19.64 & 4.84 & -14.79 & 24.42 & 11.56 & -12.86 \\
$5^{\prime}$ & 16.69 & 3.75 & -12.94 & 23.06 & 8.61 & -14.45 \\
\hline
\end{tabular}

TABle 2: Table shows OR, CI 95\%, and $P$ value calculated with logistic regression model to evaluate statistical significance of the variation between pneumatic dilation and myotomy treatment in patients with diagnosis of achalasia.

\begin{tabular}{lcccc}
\hline & $\begin{array}{c}\text { Odd } \\
\text { ratio }\end{array}$ & Confidence interval (95\%) & $\begin{array}{c}P \\
\text { value }\end{array}$ \\
\hline $\begin{array}{l}\text { Reduction > mean } \\
\text { Esophagus }\end{array}$ & 0.167 & 0.02 & 1.419 & 0.1011 \\
$\begin{array}{l}\text { Column baryta } \\
\text { height }\end{array}$ & & & & \\
$0^{\prime}$ & 0.625 & 0.093 & 4.222 & 0.6297 \\
$1^{\prime}$ & 0.625 & 0.093 & 4.222 & 0.6297 \\
$2^{\prime}$ & 0.429 & 0.062 & 2.972 & 0.3911 \\
$5^{\prime}$ & 0.9 & 0.133 & 6.080 & 0.9139 \\
\hline
\end{tabular}

and abdominal CT scan without and with intravenous $\mathrm{cm}$ may be helpful in specific, not so common cases $[9,14]$.

The barium X-ray examination allows to confirm the diagnosis and to assess the degree of esophageal dilation, the axis of the esophagus, and the presence of an associated epiphrenic diverticulum [6], the esophagus appears dilated, aperistaltic, or with uncoordinated peristaltic contractions, sometimes stuffed of food previously ingested and with the "tail mouse" characteristic appearance of the cardiac region [6].

In the early stages, the only sign may be the endoluminal stagnation of $\mathrm{cm}$, with a progressive increase in the height of the barium column until its pressure causes the forced opening and subsequent rapid emptying of the LES.

The cause for an initial reduction of inhibitory neurons in achalasia is unknown; then etiological therapies still do not exist, but only symptomatic treatments [9]. These treatments are designed to solve the lack of LES relaxation. The therapeutic approaches may be pharmacological, endoscopic, or surgical. Drug therapy is not very effective, because, even in the early stages of the treatment, the benefits can be seen only in about $2 / 3$ of the patients, with a chronic drug intake that may cause a reduction in the pharmacological effects with tolerance and addiction phenomena; the possible presence of side effects, such as low blood pressure and related headaches, has to be considered [15]. Some studies have shown a partial efficacy of calcium channel blockers and nitrated derivatives [16], but the use of these medical therapies should be reserved for those patients who cannot tolerate surgical approaches or to who refuse to use them.

Another type of treatment consists of endoscopic therapy that includes the botulinum toxin injection (BTI) and the pneumatic dilation (PD) $[17,18]$.

BTI is based on a botulinum toxin endoscopic injection in the cardia leading to an inhibition of release of acetylcholine from the myenteric plexus resulting in reduction of smooth muscle contraction of the cardiac region. The effects of a single treatment can persist for six months or more (up to 2-3 years).

The PD consists in the endoscopic introduction, through the mouth, of special dilators, on a metal guide introduced until after the cardia, with the patient maintained under sedation. The dilators consist in cylindrical balloon length of about $12 \mathrm{~cm}$ and with variable diameter $(2.5$ to $4 \mathrm{~cm}$ ), progressively positioned in the cardiac region. Once placed, it is swollen for 1 minute at 15 PSI pressure. Usually one or two dilations are sufficient to obtain a good result. In $3 \%$ of cases, however, there is a cumulative risk of incurring postoperative complications such as tearing and/or perforation of the esophagus. With this method $60-70 \%$ of good results may be obtained [19]. The surgical Heller extramucosal myotomy represents the surgical treatment of choice [20]. The intervention consists in the longitudinal section of the cardial esophageal smooth musculature for 6-7 cm; then, an antireflux Dor fundoplication is associated, protecting the esophageal mucosa from the gastroesophageal reflux. According to the available literature, good or excellent results may be obtained in up to $90 \%$ of the cases [21].

In our study, the barium X-ray examination of the esophagus (Figure 1) in patients with achalasia, performed before and after the surgical and endoscopic pneumatic treatments, has shown that the average reduction of barium column height observed in patients surgically treated was more noticeable if compared with those treated with pneumatic endoscopic dilation as well as the reduction in the esophageal caliber. The barium X-ray examination was a good test to evaluate the outcome after surgery or endoscopic dilation, being well tolerated and poorly invasive and allowing objectively defining, with a quantitative analysis of the esophageal caliber and morphology. In conclusion, the surgical treatment represents the treatment of choice of achalasia, giving better and more stable results in comparison with endoscopic pneumatic dilation reserved for patients with high operative risk and who refuse surgery; the esophagus X-ray barium study is the modality of choice in the preoperative and postoperative imaging evaluation of these patients.

\section{Conflict of Interests}

The authors declare that there is no conflict of interests regarding the publication of this paper. 


\section{References}

[1] J. E. Pandolfino and A. J. Gawron, "Achalasia: a sistematic review," The Journal of the American Medical Association, vol. 313, no. 18, pp. 1841-1852, 2015.

[2] S. P. Bowers, "Esophageal motility disorders," Surgical Clinics of North America, vol. 95, no. 3, pp. 467-482, 2015.

[3] M. I. Yasawy, "Diagnosis and treatment of achalasia patients: a ten-year review of the clinical features," Hepatogastroenterology, vol. 61, no. 134, pp. 1611-1616, 2014.

[4] A. Moonen and G. Boeckxstaens, "Current diagnosis and management of achalasia," Journal of Clinical Gastroenterology, vol. 48, no. 6, pp. 484-490, 2014.

[5] A. Dobrowolsky and P. M. Fisichella, "The management of esophageal achalasia: from diagnosis to surgical treatment," Updates in Surgery, vol. 66, no. 1, pp. 23-29, 2014.

[6] I. Gockel, V. F. Eckardt, T. Schmitt, and T. Junginger, "Pseudoachalasia: a case series and analysis of the literature," Scandinavian Journal of Gastroenterology, vol. 40, no. 4, pp. 378-385, 2005.

[7] L. Abbes, M. Leconte, R. Coriat, B. Dousset, S. Chaussade, and M. Gaudric, "Achalasia: role of endoscopic therapy and surgery," Presse Medicale, vol. 42, no. 5, pp. 814-818, 2013.

[8] G. Zuccaro Jr., "Esophagoscopy and endoscopic esophageal ultrasound in the assessment of esophageal function," Seminars in Thoracic and Cardiovascular Surgery, vol. 13, no. 3, pp. 226233, 2001.

[9] A. R. Choi, N. R. Chon, Y. H. Youn, H. C. Paik, Y. H. Kim, and H. Park, "Esophageal cancer in esophageal diverticula associated with achalasia," Clinical Endoscopy, vol. 48, no. 1, pp. 70-73, 2015.

[10] S. Maeyaert, J. Pringot, J. L. Samain, L. Ghijseling, and P. Matthys, "Cancer of the oesophagus in achalasia," Journal Belge de Radiologie-Belgisch Tijdschrift voor Radiologi, vol. 96, no. 1, p. 46, 2013.

[11] M. Ramirez and M. G. Patti, "Changes in the diagnosis and treatment of achalasia," Clinical and Translational Gastroenterology, vol. 6, no. 5, article e87, 2015.

[12] J. E. Richter, "High-resolution manometry in diagnosis and treatment of achalasia: help or hype," Current Gastroenterology Reports, vol. 16, no. 12, article 420, 2014.

[13] M. Müller, "Impact of high-resolution manometry on achalasia diagnosis and treatment," Annals of Gastroenterology, vol. 28, no. 1, pp. 3-9, 2015.

[14] J. E. Pandolfino and A. J. Gawron, "Achalasia: a systematic review," Journal of the American Medical Association, vol. 313, no. 18, pp. 1841-1852, 2015.

[15] M. F. Vela, "Management strategies for achalasia," Neurogastroenterology \& Motility, vol. 26, no. 9, pp. 1215-1221, 2014.

[16] Z. H. Wen, E. Gardener, and Y. P. Wang, "Nitrates for achalasia," Cochrane Database of Systematic Reviews, no. 4, Article ID CD002299, 2002.

[17] D. A. Katzka and D. O. Castell, "Review article: an analysis of the efficacy, perforation rates and methods used in pneumatic dilation for achalasia," Alimentary Pharmacology \& Therapeutics, vol. 34, no. 8, pp. 832-839, 2011.

[18] Z. Ramzan and A. B. Nassri, "The role of Botulinum toxin injection in the management of achalasia," Current Opinion in Gastroenterology, vol. 29, no. 4, pp. 468-473, 2013.
[19] M. E. Allaix and M. G. Patti, "Endoscopic dilatation, heller myotomy, and peroral endoscopic myotomy: treatment modalities for achalasia," Surgical Clinics of North America, vol. 95, no. 3, pp. 567-578, 2015.

[20] G. Torres-Villalobos and L. A. Martin-del-Campo, "Surgical treatment for achalasia of the esophagus: laparoscopic Heller myotomy," Gastroenterology Research and Practice, vol. 2013, Article ID 708327, 5 pages, 2013.

[21] A. Kolinioti, B. Kayani, C. Skouras, A. Fovos, O. Aziz, and E. Zacharakis, "Does laparoscopic Heller's myotomy provide superior results compared to endoscopic dilatation for oesophageal achalasia?" International Journal of Surgery, vol. 11, no. 3, pp. 238-243, 2013. 


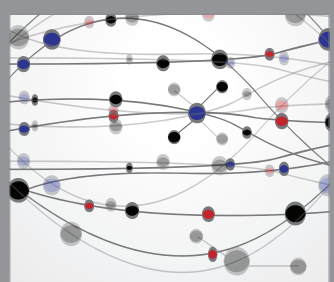

The Scientific World Journal
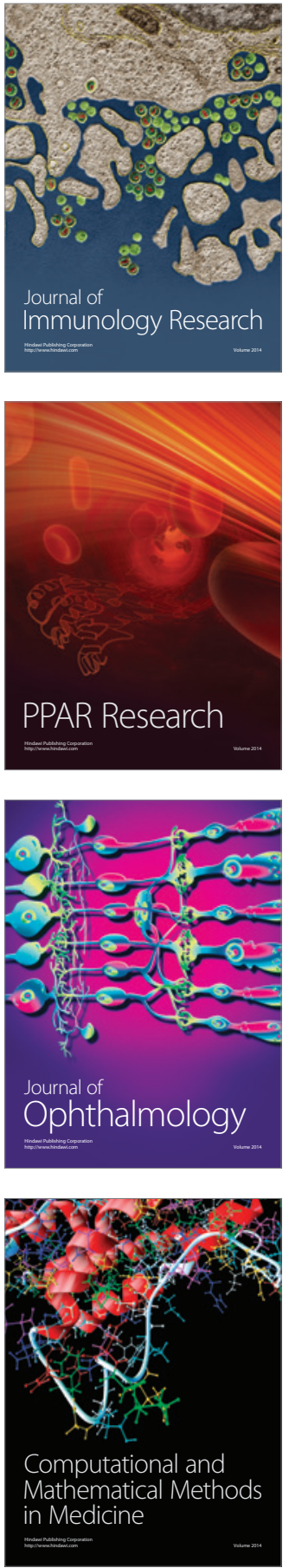

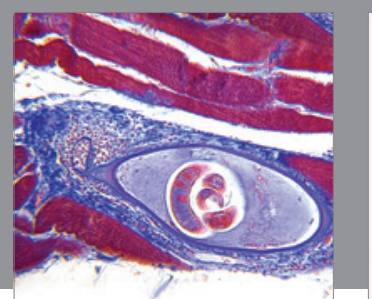

Gastroenterology Research and Practice

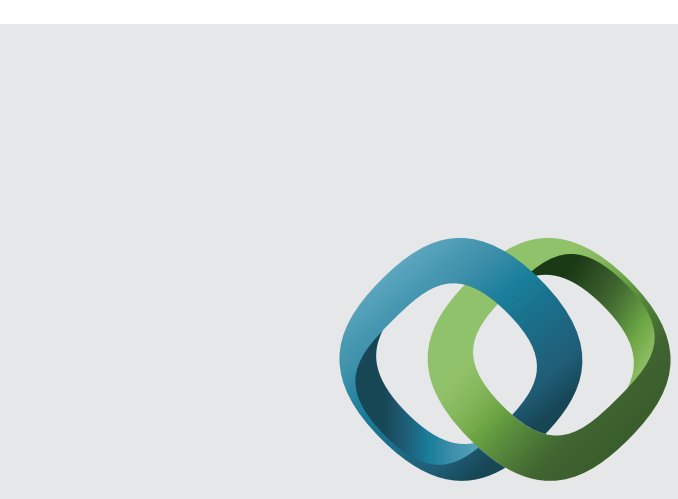

\section{Hindawi}

Submit your manuscripts at

http://www.hindawi.com
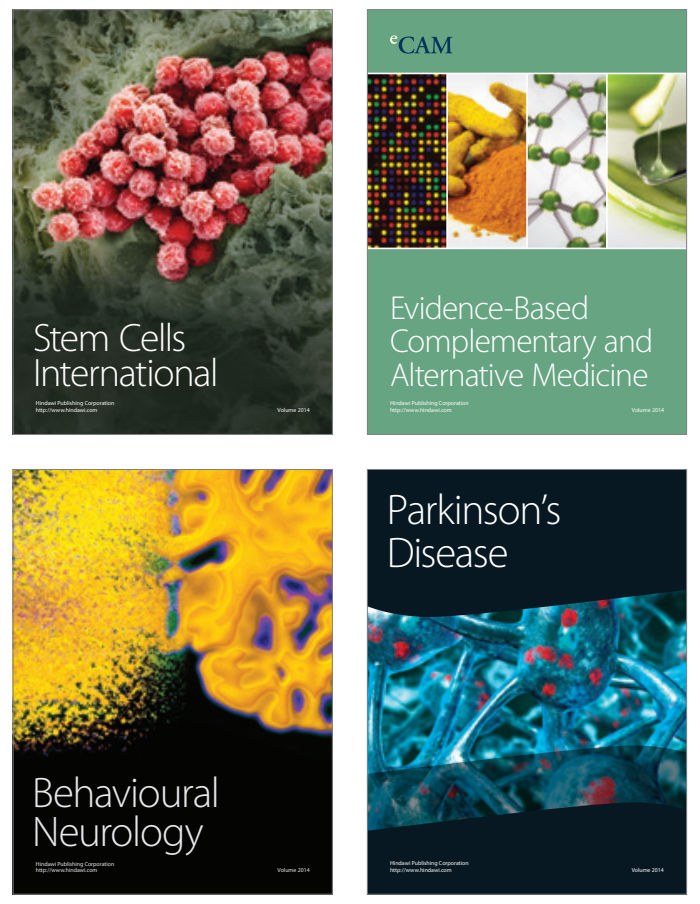
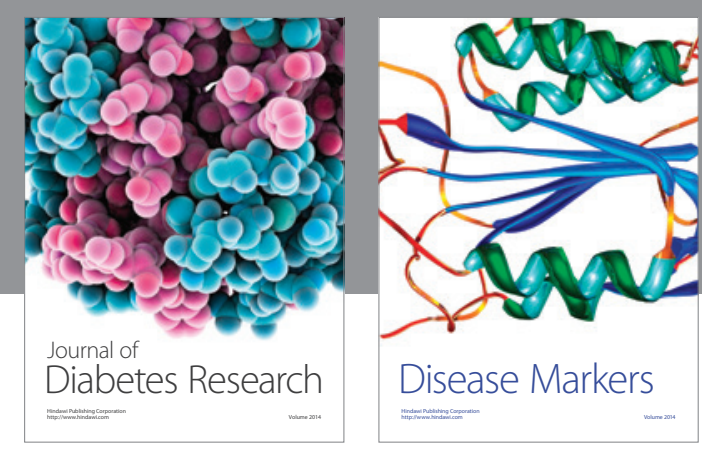

Disease Markers
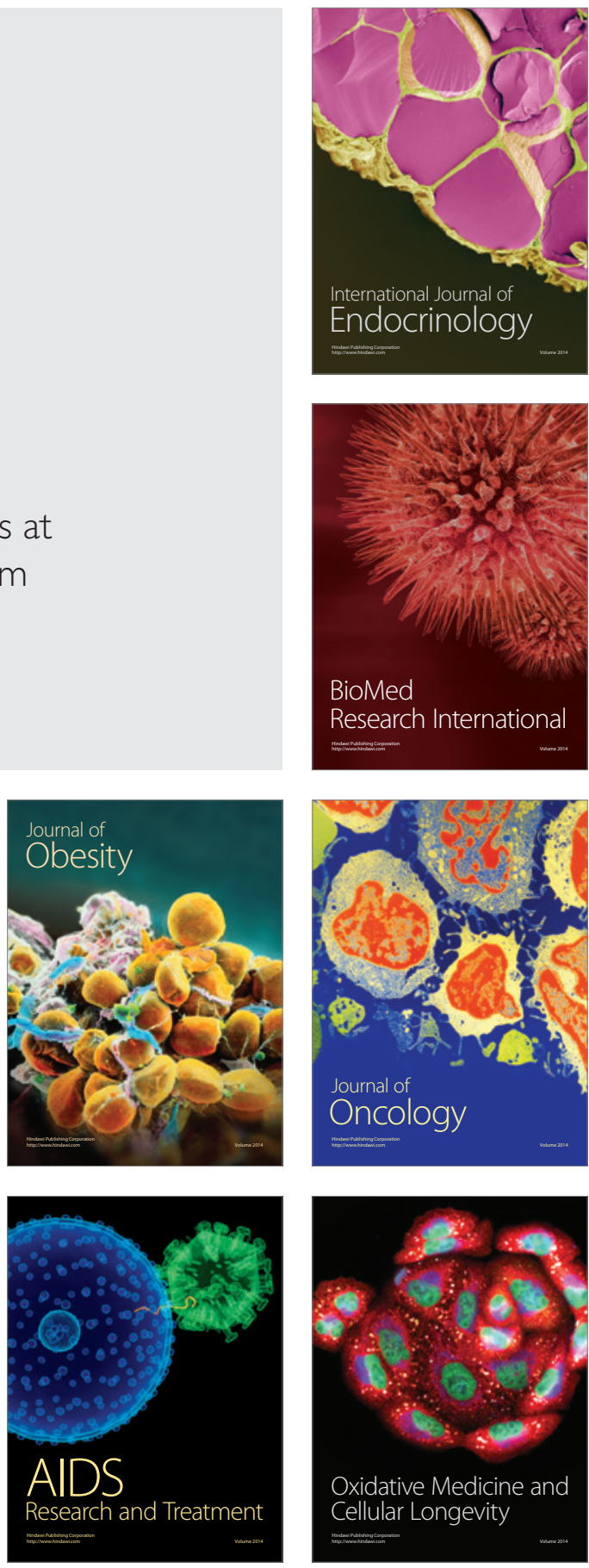